\title{
El proceso enseñanza - aprendizaje - evaluación (PEAE) una didáctica universitaria
}

\author{
Yaćhana-yaćhachina puliynin (PEAE) huk \\ yaćhanap hatun yaćhaywasićhu
}

\section{Kara okanta ogomeantagantsi- ogotagantsi- monkaratagantsi(PAE) kantagero kara ogomentosipongokë aroka aregmaro.}

Recibido: 11 abril 2020 Corregido: 23 mayo 2020 Aprobado: 15 junio 2019

Oscar Gutiérrez Huamaní

Nacionalidad: Peruana / Universidad Nacional de San Cristóbal de Huamanga Correo: oscar.gutierrez@unsch.edu.pe /ORCID: https://orcid.org/oooo-0002-8070-0108

Delia Ayala Esquivel

Nacionalidad: Peruana / Universidad Nacional de San Cristóbal de Huamanga Correo: delia.ayala@unsch.edu.pe / ORCID: https://orcid.org/oooo-0003-4715-291X

\section{Resumen}

El mundo prioritariamente fue visto con el paradigma de la simplicidad que mutila y sesga al fenómeno o realidad; esto nos invita orientar nuestra visión hacia el pensamiento complejo. El proceso educativo entendido como una unidad indivisible, simultánea y permanente, reconoce a la enseñanza, aprendizaje y evaluación como un único proceso complejo. El PEAE como didáctica universitaria permite consolidar el desarrollo de las competencias específicas de una carrera profesional, empleando el laboratorio educativo para canalizar la consecución de desempeños, con una visión compleja y sistémica, entendiendo y asumiendo los aspectos ontoepistemológicos de la educación universitaria.

\section{Palabras clave:}

laboratorio, pensamiento complejo, enseñanza, aprendizaje, evaluación.

\section{Lisichiku limaykuna:}

Lulaywasi, allip umachakuy, yaćhachi, yaćhana, tapukuy.

\section{Nibarintsipage katingatsaro:}

Oigotagetiro, kengagantsi komëtaro, ogometagantsi, monkaratagantsi ogotaneegi. 


\section{Migration of The People of Nuñungayocc, of the District of Palca-Huancavelica}

\begin{abstract}
The world was seen primarily with the paradigm of simplicity that mutilates and divide the phenomenon or reality; this invites us to orient our vision towards complex thinking. The educational process understood as an indivisible, simultaneous and permanent unit, recognizes teaching, learning and evaluation as a single complex process. The PTLE as a university teaching allows consolidating the development of the specific competences of a professional career, using the educational laboratory to channel the achievement of performances, with a complex and systemic vision, understanding and assuming the onto-epistemological aspects of university education.
\end{abstract}

\author{
Keywords \\ laboratory, complex \\ thinking, teaching, \\ learning, evaluation.
}

\section{A migração dos moradores de ñuñungayocc do distrito de Palca-Huancavelica}

\section{Resumo}

O mundo foi visto prioritariamente com o paradigma da simplicidade que mutila e distorce o fenômeno ou a realidade; isso nos convida a orientar nossa visão em direção a pensamentos complexos. O processo educativo, entendido como uma unidade indivisível, simultânea e permanente, reconhece o ensino, a aprendizagem e a avaliação como um processo único e complexo. O PEAE como didática universitária permite consolidar o desenvolvimento das competências específicas de uma carreira profissional, utilizando o laboratório educacional para canalizar a realização de desempenhos com uma visão complexa e sistêmica, entendendo e assumindo os aspectos ontoepistemológicos da educação universitária.

\section{Palavras-chave:}

Laboratório, pensamento complexo, ensino, aprendizagem, avaliação.

\section{Datos de los autores}

Oscar Gutiérrez Huamaní es docente e investigador en ciencias de la educación, actividad física y salud. Doctor en Ciencias de la motricidad por la Universidad Estatal Paulista "Júlio de Mesquita Filho" (UNESP), Ciudad de Rio Claro, Sao Paulo, Brasil. Magister en Educación Física por la Universidad Mayor de San Marcos del Perú

Delia Ayala Esquivel es docente e investigador en Ciencias de la Educación, actividad física y salud. Doctora en Educación por la Universidad César Vallejo del Perú. Magister en Educación, con mención en Docencia y Gestión Educativa por la Universidad César Vallejo del Perú 


\section{Introducción}

En un contexto de despunte de la economía, la educación es vista como la impulsora del desarrollo; surgiendo el concepto de desarrollo como "crecimiento económico" creando la dualidad educación-economía y economía-educación (España, 2017). Este contexto obliga a las Universidades a formar profesionales competentes que respondan a las exigencias actuales con eficiencia, eficacia y efectividad, brindando el desarrollo de competencias específicas de cada carrera profesional. Estableciéndose como una posibilidad, la formación a través del trabajo de las universidades y las empresas (educación-economía). Las características de las diferentes especialidades, la multiplicidad de conocimientos, especificidad de competencias profesionales y los diferentes niveles de formación: pregrados, maestrías, doctorados y posdoctorados, hace que el proceso educativo en las universidades sea compleja.

Las universidades peruanas están travesando el proceso de autoevaluación, licenciamiento, acreditación, en busca de desarrollo y competitividad, teniendo estándares que cumplir (SINEACE, 2017), siendo el desarrollo del currículo por competencias el estándar 11, del factor 4 Proceso enseñanza aprendizaje, de la dimensión 2 Formación integral (SINEACE, 2016), uno de los estándares a tener en cuenta. Esta efervescencia por las competencias en las universidades, está exigiendo la construcción, desarrollo y evaluación de los currículos por competencias, con estrategias adecuadas para diferentes escenarios y carreras de formación universitaria (jurídico, clínico, educativo, entre otros). Este panorama se da en un entorno problemático:

* Muchas de las universidades aún tienen currículos centrados en contenidos.

* No existe una teoría unificadora las competencias con escasa experiencia curricular basado en este enfoque (Moreno, 2012).

* El examen de admisión y examen final de asignatura son estrategias más utilizadas en las universidades, estos instrumentos no evalúan competencias (Obando, 2014), además la falta de uso de las estrategias para desarrollar competencias, y el considerar a la evaluación como un proceso aislado del acto educativo, siguen conviviendo en las universidades peruanas.

Este contexto nos da oportunidad de reflexionar sobre ¿Cómo visualizamos al proceso educativo universitario y por qué lo dividimos en momentos distintos: Enseñanza - aprendizaje y a la evaluación como otro momento aislado del proceso educativo? ¿Existen estrategias para trabajar en las universidades como proceso enseñanza-aprendizaje-evaluación (PEAE), como una respuesta a la exigencia de un currículo por competencia?

Partamos reflexionando sobre el proceso educativo universitario y la necesidad de implementar estrategias que desarrollen competencias. Nuestro pensamiento fragmentado del conocimiento nos hacer ver al proceso educativo, es decir a la enseñanza, aprendizaje y evaluación como elementos diferentes y específicos. Pero en la actualidad es necesario visualizar hacia una postura sistémica, compleja, transdisciplinar y autorreflexiva del conocimiento, “... no se puede cercenar el conocimiento y condicionarlo a verdades o leyes universales..." (Torres \& Lugo, 2020, P. 2). En ese sentido el proceso educativos visto con el pensamiento complejo busca el conocimiento multidimensional, en donde "la totalidad es la novedad" (Morín, 2011); es decir PEAE, "como un procesos en busca de un saber no parcelado, no dividido, no reduccionista” (Morín, 2011). 


\section{Las competencias en el ámbito universitario}

El peligro del currículo por competencias es la necesidad de cumplir estándares y criterios de validez y la satisfacción de competencias establecidas, para demostrar la competencia profesional en una determinada carrera, por lo que todo profesional debe conseguir dichos estándares, desde el proceso de selección del postulante, formación y evaluación de esas competencias. Esto podría ser tomado como un fenómeno discriminatorio, por ejemplo si en una admisión universitaria a una determinada carrera, el estándar obliga a tener una característica o cualidad física específica (talla $170 \mathrm{~cm}$ o no tener discapacidad física - dedos incompletos de la mano) el postulante que no cumple este estándar no será admitido.

La teoría de la competencia proviene en parte de las ciencias del comportamiento (la sociología, la psicología, la economía, la antropología y la pedagogía) en función a las áreas de la "Motivación humana", "Rasgos de personalidad" y la "Auto-imagen". Van más allá de ser simples recomendaciones organizativas en el contexto empresarial. El término "competencia" o "competencias" tienen la connotación de habilidad, capacidad, idoneidad, de acuerdo a una serie de conductas observables, básicamente en un entorno laboral y profesional (Guerrero \& Jiménez, 2011).

La competencia es la capacidad de responder a las demandas, realizando las tareas de forma adecuada combinando habilidades prácticas, conocimientos, motivación, valores éticos, actitudes, emociones, así como otros componentes sociales y comportamentales empleados conjuntamente para lograr una acción eficaz. Las competencias desde la vista puramente económica, impulsa la productividad y la competitividad en el mercado; minimiza el desempleo mediante el desarrollo de una mano de obra adaptable y calificada en un mundo dominado por la competencia global (OECD, 2002).

La competencia, es la capacidad y habilidad de utilizar los saberes más adecuados e idóneos. La competencia se exterioriza a través de la capacidad de resolver problemas con las herramientas conceptuales y procedimentales adquiridas, está ligada a una acción concreta (desempeño) que se comprueba concretamente con la competencia adquirida. La educación por competencias conduce a un sistema de estructura cerrada: competencias básicas y específicas (Salinas, Costillas (Cood.), 2007; Ferrer, 2007; Álvarez, 2008).

El positivismo tecno-funcionalista con este enfoque, mezcla los conceptos, ideas y propuestas para plantear una educación por competencias, dando un papel destacado a competencia convirtiéndolo en contenido, con una retórica que crea la sensación de una visión amplia y abierta de la educación; dando la impresión que de todo va a funcionar de un modo perfecto, pero no podemos asegurar una mejora del sistema educativo por sí mismas, pretendiendo formar seres homogéneos, homologables bajo el concepto de competentes (Álvarez, 2008; Salinas \& Costillas (Cood.), 2007). Por lo que, optar por un currículo universitario por competencias, aparentemente mejorará por completo la educación y formación profesional; pero en la realidad no es así, ya que los docentes necesitamos de empoderarnos de estrategias que desarrollen desempeños profesionales específicos, y no una enseñanza de contenidos teóricos.

La expresión "enseñanza centrada en competencias" encierra más una aspiración con clara necesidad de cambio, evaluando la existencia de las condiciones del sistema educativo, encubriendo las concepciones pragmático-funcionales con interés inspirado en la formación profesional y técnica para el mercado laboral (Álvarez, 2008). Aquí es necesario una la reflexión 
sobre qué formación universitaria debemos aspirar: una formación técnico profesional o una formación humanística profesional, y no sólo estandarizada por desempeños que acrediten una determinada carrera profesional. La necesidad de que el ser humano mantenga su diversidad frente a una estandarización es vital; el arte, el deporte, la cultura, la música no debían de ser estandarizados.

Sin duda alguna el reto de asumir el proceso educativo universitario por competencia desde la perspectiva de la complejidad, nos obliga a visualizar el PEAE como un reto, en el que necesariamente se integren estos aspectos así como tener en cuenta una visión onto-epistémica. Con este afán abordaremos en forma desarticulada los procesos de enseñanza, aprendizaje y evaluación para luego plantearlos como un proceso único.

\section{La enseñanza universitaria}

La formación didáctica de los docentes universitarios debe estar orientado a permitir la transferencia y desarrollo de las habilidades del pensamiento crítico, inferencia, toma de decisiones, curiosidad intelectual, sentido crítico, acucia de la duda y pensamiento creativo; ello propiciará desarrollar en los estudiantes además del conocimiento propio de cada asignatura, las competencias necesarias para su aplicación en cualquier contexto.

La didáctica universitaria ha de desarrollarse dialógicamente, despertando en el estudiante interés y atención en los contenidos tratados; desde ahí profundizar otros contenidos, respetando el interés del estudiante, salvaguardando las competencias fundamentales de cada especialidad. Interés que hay que buscarlo o incluso rastrearlo con preguntas que sirvan de guion a la clase del día, situando al estudiante en el espejo de su propio bagaje e integrar esos aprendizajes (Coronel, 2007).

La enseñanza debe propiciar la aplicación de procesos mentales para el manejo del conocimiento, basado en un modelo de aprendizaje activo, significativo y dirigido al desarrollo de las potencialidades del ser humano en un ambiente de apertura mental, de interacción, participativa y respeto a la diversidad (Rojas, 2009).

\section{El aprendizaje en la universidad}

El aprendizaje en el cerebro se produce con un despliegue de neurotransmisores que permite recibir, seleccionar y priorizar la información, creándose redes neurales que se almacenan por la frecuencia de su uso. Cuando el aprendizaje es placentero y motivante la amígdala segregará mayor dopamina, lo que fortalecerá la capacidad de aprender con facilidad, en un estado emocional positivo el hipocampo creará nuevos recuerdos y dará la posibilidad de la neurogénesis, siendo importantes las emociones positivas para el aprendizaje. Esta situación también permite liberar otros neurotransmisores como la acetilcolina que mejora el estado de alerta, la memoria y otras funciones ejecutivas en la corteza prefrontal. La memoria (corto y largo plazo) tiene implicaciones en la educación, siendo fundamental para relacionar con lo que ya se sabe generando nuevos pensamientos (Rojas, 2009).

Las personas aprendemos no porque se nos transmita la información, sino porque construimos nuestra versión personal de la información. No estamos controlados por el componente cognitivo, sino por el componente emotivo, el agresivo en particular (Levi-Montalcini, 
2005). El aprendizaje en la universidad debe desarrollar la capacidad activa y voluntaria de construir juicios independientes (acto mental) y la capacidad de ir reconociendo las propias capacidades, habilidades o conductas, como persona y como futuro profesional propiciando el logro de actitudes y valores. El aprendizaje profundo tiene una motivación intrínseca por comprender más allá del recordar, basada en el interés por aquello que se trabaja, es decir comprender, relacionar, construir un discurso propio y relevante; y resolver problemas de forma creativa (Salinas \& Cotillas, 2007; Rojas, 2009).

\section{La evaluación en la universidad}

La evaluación en la universidad permite el desarrollo de competencias profesionales, convirtiéndose en un mecanismo de retroalimentación de los desempeños del estudiante, elaborando un juicio sobre el valor o mérito de algo, con dos etapas: recogida de evidencias y aplicación de ciertos criterios de calidad sobre esas evidencias que nos permita la estimación del aspecto evaluado. Una evaluación bien razonada y justificada, puede ayudar al estudiante a entender la valía de su trabajo y actuación en relación a los criterios de evaluación y en relación a los trabajos y actuaciones de otros, por lo que la viabilidad es una de las condiciones básicas de toda evaluación, para establecer la selección de evidencias y la aplicación de los criterios sobre dichas evidencias constituyéndose en un proceso razonablemente posible (Salinas \& Cotillas, (Cood.) 2007).

La evaluación parte de una idea de la docencia universitaria, determinada por una concepción de la enseñanza universitaria, garantizando la impartición cabal de las competencias fundamentales de cada especialidad (Coronel, 2007). La auténtica evaluación no solo busca el "éxito académico" (notas, calificaciones) sino, la trascendencia educativa, con el acompañamiento del docente en el proceso de construcción de aprendizaje. Esta debe fomentar debates, el pensamiento divergente y las respuestas múltiples con un carácter sistemático e integrador del proceso educativo, al servicio de quien aprende y de quien enseña como una función formativa; siendo dinámico, abierto, continua, global, integradora, vertebrador, dinamizador, contextualizado e individualizado. Evaluar para corregir errores a tiempo y explicar las causas que los motivan después del aprendizaje (Álvarez, 2008; Castillo \& Cabrerizo, 2010).

\section{El proceso educativo: Proceso Enseñanza- Aprendizaje-Evaluación (PEAE)}

Partamos por intentar entender la complejidad. La complejidad en una primera mirada es un tejido (una tela contemporánea) constituido de hilos heterogéneas y asociadas inseparablemente: ella coloca la paradoja de lo uno y de lo múltiple. En un segundo momento, la complejidad es efectivamente el tejido de acontecimientos, acciones, interacciones, retroacciones, determinaciones, posibilidades, que constituyen nuestro mundo fenómico. Mas entonces la complejidad se presenta con un trazo inquietante del enmarañado, de inextricable, del desorden, de la ambigüedad, de la incerteza (Morín, 2011).

El hecho de fragmentar al proceso educativo (enseñanza-aprendizaje y evaluación) para su estudio, dando una mirada focalizada hacia un solo aspecto o ángulo, genera sesgo distorsionando el fenómeno, por ello intentaremos entender al PEAE como un proceso único y transdisciplinar. El PEAE es un reto que procura la construcción de una nueva visión, a partir 
de ser humano, sin obviar el ambiente y entorno, como un sistema que se auto-organiza y evalúa, que redefine y reconstruye el proceso educativo en un fenómeno inacabado, auto-organizado y complejo. Sin negar los avances de todos los pedagogos y sus aportes didácticos, sino reflexionando sobre el proceso educativo en sí, con el protagonismos de los sujetos que intervienen en la acción educativa renovando el conocimiento de la didáctica universitaria con una visión compleja e integral, incorporando "los principios revolucionarios de la microfísica, la cibernética, la teoría general de sistemas, la redefinición del hombre, desde todos los campos del saber y la necesidad de generar un conocimiento eco-sistemático, donde el ambiente aparece como un elemento innegable" (Torres \& Lugo, 2020).

La unidad y la transdisciplinariedad del conocimiento científico permite integrar cada área, de manera tal que se elimine la hendidura y compartimentalización del saber, reconoce visualizar el conocimiento como una red interconectada, desbordada de un sinfín de fenómenos o sucesos biológicos, sociales, psicológicos, ambientales, físicos, entre otros, que dependen uno del otro (Torres \& Lugo, 2020).

El positivismo lógico excluye al sujeto como agente observador para estudiar el objeto, bajo la premisa de mantener la objetividad disciplinar (Torres \& Lugo, 2020). Entonces será correcto estudiar a la enseñanza, aprendizaje y evaluación sin la participación de los sujetos que intervienen en el proceso educativo, y centrarse sólo en el aspecto metodológico (objeto de estudio) o dar un mirada onto-epistémica al sujeto quien es el directo generador del conocimiento. En ese sentido es necesario entender que el PEAE mantiene un dualismo indisoluble entre el sujeto onto-epistémico y el proceso educativo en sí, engranándose sin mutilaciones, ni reducir o segmentar el hecho educativo.

La ontología refiere también a las personas individuales, grupos, la organización en su conjunto, o las relaciones interorganizativas en las que reside e interacciona el conocimiento que comparten con cuatro etapas: socialización, externalización, combinación e internalización. Y la epistemología relacionada al Dasein (la propia existencia) llevando lo tácito a lo explícito por la reflexión, donde el hombre ya no es abstracto sino tiene existencia real, concreta, histórica y es ontológico por la compresión del ser (Guanipa, 2017).

El Proceso Enseñanza-Aprendizaje-Evaluación (PEAE) debe ser entendido como un proceso único y no parcelado, en el que los sujetos educativos interactúan en forma permanente, considerando los aspectos bio-psico-fisico-sociocultural que acompañan a este proceso con un enfoque sistémico y multidimensional. EI PEAE aplica los procesos mentales para propiciar el manejo del conocimiento involucrando e integrando cada uno de sus componentes en forma sistémica, siendo capaz de transferirlo a diversas situaciones, ámbitos y áreas; aprendiendo tanto de los errores como de los aciertos.

En el paradigma de la simplicidad se divide el proceso educativo en: enseñanza como un proceso, el aprendizaje como otro proceso y la evaluación como un proceso complementario. EI PEAE desde el pensamiento complejo nos dará la posibilidad de plantear nuevas forma didácticas que permitan mejorar la educación universitaria, promoviendo al estudiante universitario resolver diversos estados problemáticos a otro deseado. EI PEAE sea esta informal o formal, es un proceso único en la que intervienen inseparablemente la enseñanza-aprendizaje-evaluación y todos los aspectos concomitantes. 


\section{Proceso enseñanza-aprendizaje-evaluación (PEAE)}

\section{Mirada onto-epistémica}

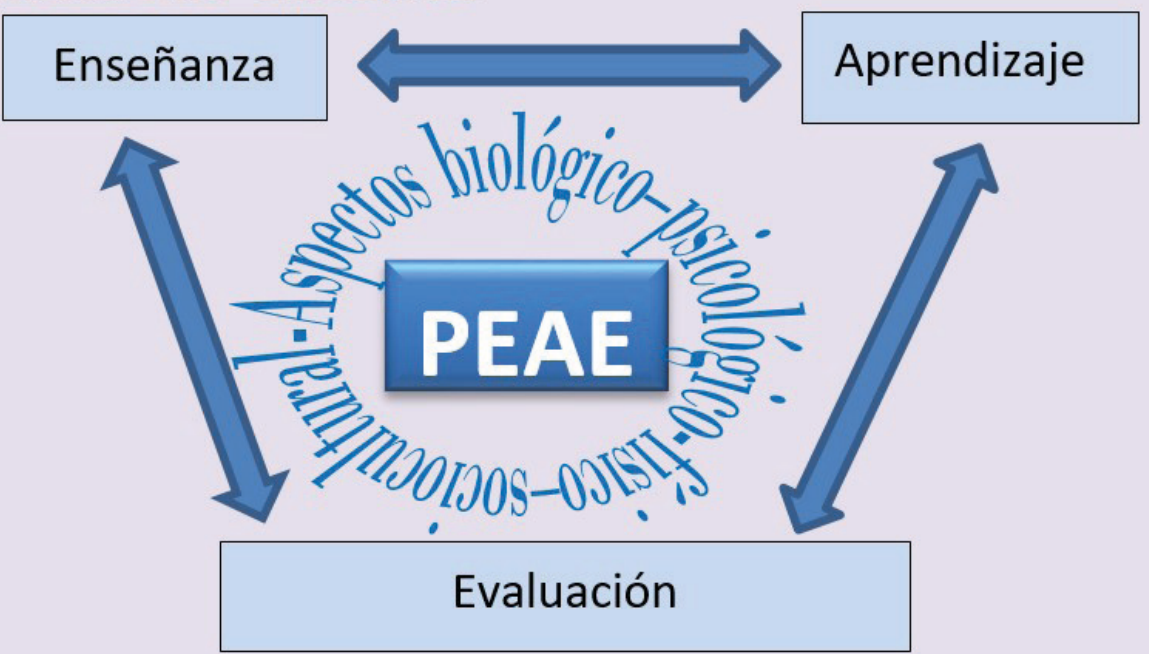

Enfoque sistémico, complejo y multidimensional

Fuente. Elaboración propia

La disociación del PEAE en los procesos de enseñanza-aprendizaje y de evaluación; subvalúa a la evaluación ocasionando en el docente la creencia de "pérdida de tiempo en la avaluación". En realidad la evaluación está inmersa en todo momento, emitiendo juicios de valor, no sólo a los procesos dados sino a todo el entorno y factores que suscitan en el acto educativo. Por ello el PEAE debe darse en un clima educacional agradable, atractivo, multisensorial, multipropósito, estructurada y organizada propiciando el aprendizaje activo, mediante participación e interacción con los demás agentes educativos.

EI PEAE debe ser el acto que despierte el interés de descubrir, explorar, apropiación de saberes, habilidades, destrezas y competencias; involucrando en forma permanente la evaluación orientado a regular todo el proceso. Enfatizando que en ningún momento existe un proceso aislado, sino es simultánea, permanente y continuo como un proceso dialéctico indivisible.

La educación superior tiene que ver con que el educando gane capacidad y autoconfianza para inquirir, desafiar, crear e innovar en la asignatura, y no es acumular una serie de hechos o conocimientos para soltar por escrito, para olvidarlos al día siguiente. Se requiere de docentes y educandos comprometidos en actividades que resulten satisfactorias, disfrutables, desafiantes y apasionantes (Chomsky, 2013). La educación actual está orientado para superar obstáculos y no para aprender, entender y explorar los conocimientos y competencias. EI PEAE debe inspirar e incentivar a los estudiantes a descubrir por sí mismos, a cuestionar cuando no estén de acuerdo, a buscar nuevas y variadas alternativas, a revisar los grandes logros del pasado y aprender de ellos. 
El sistema universitario debe añadir las nuevas tecnologías, el internet es una poderosa herramienta de trabajo, de estudio y del ocio. Los programas de moodle, mooc, camilo, etc., son una posibilidad para el PEAE, porque da la apertura a la educación a distancia (E-learning), y más en situaciones de aislamiento social que nos tocó vivir. Así mismo, las universidades necesitan asumir la gestión de conocimiento considerando al PEAE como un único y complejo proceso.

La didáctica universitaria para desarrollar competencias genéricas y específicas pueden emplear algunos métodos: el aprendizaje basado en problemas (ABP), la enseñanza en pequeños grupos, el sistema tutorial, la enseñanza en laboratorios, la investigación independiente con supervisor, el debate, la casuística, la cartografía conceptual (mapas mentales, lluvia de ideas, mapas conceptuales), los proyectos, lecturas entre otros (Obando, 2014). Estos métodos pueden ser adoptados con una visión compleja, sistémica y de gestión del conocimiento.

Todo planteamiento teórico debe generar procesos prácticos y aplicables llevados a la acción del PEAE, para generar habilidades y desempeños (facultad de una persona). Asumiremos el PEAE sin considerar la enseñanza (métodos de enseñar), aprendizaje (métodos de aprender) y a la evaluación (método de valorar y medir) como objeto de la educción - sino al ser humano como actor del proceso educativo (PEAE).

El PEAE puede operativizarse con: 1) El modelo casuístico y ABP que contribuyen de forma significativa y positiva, capacitando a los futuros profesionales a tomar decisiones, convirtiendo en los sujetos activos de su aprendizaje con la participación en el proceso de construcción del conocimiento y desarrollo de competencias; llevando a los estudiantes a la reflexión sobre cuestiones y casos reales de su futura práctica profesional. La casuística y ABP guardan una estrecha relación complementándose en la solución de un problema como estrategia para determinados contextos (Azevedo, et al., 2017; Obando, 2014). Haciéndose importante en las universidades el uso de estrategias combinadas en el PEAE para el desarrollo de competencias profesionales; estas metodologías permiten generar desempeños como proceso único enseñanza-aprendizaje-evaluación, ya que se produce en todo momento sobre el trabajo educativo de los estudiantes, quienes aprenden tanto de los aciertos como de los errores. 2) El laboratorio educativo como una propuesta directa que antecede a la práctica profesional, desarrollando competencias de la vida profesional o de la vida productiva en la empresa. EI PEAE a través de los laboratorios educativos combina el saber, el hacer y el saber ser con los demás, por entrar en contacto a un ambiente parecido a su campo de acción (laboral), con el desempeño adecuado en situaciones propias de su práctica profesional, combinando los conocimientos, habilidades y actitudes, en los que se puede generar desempeños de manera eficiente, eficaz, efectivo y productiva.

El éxito del PEAE en el laboratorio educativo experimental tiene varios factores confluyentes, como la existencia de grupos reducidos y con atención personalizada, buena programación, seguimiento de la labor realizada por el estudiante y calificaciones basadas en trabajos presentados por los estudiantes sobre la labor que allí realizan, el carácter continuo y retroactivo de la verificación. El extrapolar la aplicación del laboratorio educativo en materias con un carácter más teórico, permitiría el trabajo personalizado a través de tutorías, creando grupos reducidos de trabajo, talleres en que los estudiantes emprendan la resolución de ejercicios y problemas con la supervisión de los docentes. El trabajo en el laboratorio permite al docente el conocimiento de la capacidad, interés y evolución de los estudiantes a lo largo de todo el proceso, en cada una de las prácticas, con evaluación continua través de la libreta y memoria de laboratorio, y al estudiante realizar el trabajo experimental desarrollando habilidades cooperativas mejorando la formación académica. La corrección del trabajo de los estudiantes al finalizar cada práctica, 
puede ser discutido entre el docente y los estudiantes. Es necesario la creación de la infraestructura organizativa, inspirada en la experiencia de los laboratorios educativos, que permita a los docentes y estudiantes incorporarse al escenario laboral experimental propuesto (Ferrer, 2007). La experiencia de contar con un Laboratorio educativo experimental (Laboratorio de Actividad Física y Salud-UNSCH), nos permite señalar que en este entorno se pueden llevar el PEAE como un único proceso, ofreciendo una educación científica, tecnológica y práctica más próxima y rigurosa al ejercicio profesional, con un trabajo continuo con mayor sintonía a su formación en un entorno productivo, económico, cultural y social, conociendo los aspectos onto-epistemológicos de los miembros del laboratorio.

El laboratorio educativo experimental permite trabajar bajo un concepto sistémico y complejo llevando el PEAE hacia la gestión del conocimiento. Guanipa (2018) señala la creación del nuevo conocimiento a través del espiral que se forma en las etapas de Socialización, Externalización, Combinación e Internalización (SECI) que forman las características de clima organizativo y convergencia en valores y objetivos.

El modelo SECI produce un PEAE en el que el laboratorio educativo experimental apertura: La socialización, con el acompañamiento de los estudiantes de años superiores que comparten experiencias, sentimientos, emociones, modelos mentales y conocimiento removiendo barreras entre cada miembro del laboratorio. La externalización, que permite articular el conocimiento tácito en conocimiento explícito, práctico y aplicado, combinando la intuición y la razón en la misma experimentación. La combinación en la que se sistematiza los conocimientos que tenían los miembros del laboratorio con nuevos conocimientos y la producción o propuesta de la experimentación. La internalización en el que el estudiante incorpora el nuevo conocimiento al que ya poseía, buscando nuevas formas de generar nuevos conocimientos y experimentos comenzando un nuevo ciclo. El laboratorio permite un PEAE a través de una práctica refinada de experiencia, fruto de actividades operativas que aplica en sus actividades semestrales.

El reto epistemológico del PEAE en el laboratorio educativo experimental, se concreta con la relación dual sujeto-objeto, en el que el mismo sujeto se percata del saber generado, con la interpretación de la experiencia sistematizándolo (categorización) por inferencias de la mente humana. El aspecto ontológico del PEAE comprende justamente al ser humanos individual, grupal y sociocultural capaz de producir conocimiento en su interacción. La transdisciplinariedad rompe el estereotipo de separar el conocimiento tácito (teorías) de la cultura, ya en el laboratorio se aterrizan esos conocimientos a la experimentación práctica con todos sus componentes (biológico, psicológico, físico, moral, ecológico, cultural, social y político) reales de fenómeno estudiado. Esta forma de educar, a través del PEAE en un laboratorio permite aprender a conocer, aprender a hacer, aprender a convivir y aprender a ser bajo una concepción compleja. El trabajo del PEAE a través de laboratorio permite un acompañamiento (evaluación) estableciendo relaciones recíprocas en el desarrollo de las competencias no en forma nuclear por disciplinas o asignaturas, sino de un consenso organizado de competencias (específicas de la carrera) esperadas en su desempeño específico.

El sujeto que aprende simultáneamente evalúa: discrimina, valora, critica, opina, razona, fundamenta, decide, enjuicia, argumenta, adopta, entre lo que tiene un valor y aquello que no tiene valor para él. Si la evaluación no se realiza como fuente de aprendizaje (autorregulación constructiva), queda reducida a la aplicación de técnicas, confundiéndose con el instrumento, con el examen, con el resultado final separado del proceso educativo (Álvarez, 2008). 
Finalmente el PEAE debe tomar en cuenta las Tecnologías de la Información y la Comunicación (TIC's), que crean la posibilidad de la extensión en espacio y tiempo de la acción educativa. Cambiar la educación superior implica cambiar el cómo mensuramos (evaluamos) los desempeños.

\section{Conclusiones}

El Proceso-Enseñanza-Aprendizaje-Evaluación (PEAE) podría consolidar las competencias específicas de cada carrea profesional a través de los laboratorios educativos experimentales teniendo en cuenta los dominios ontológico, epistemológico, conceptual y metodológico; sustentados desde la complejidad y el enfoque sistémico de la educación. Las carreras profesionales deben implementar laboratorios educativos considerando las competencias específicas a lograr en cada carrera profesional. Los docentes universitarios deberían experimentar el PEAE como una forma didáctica, emprendiendo la conformación de sus laboratorios educativos en función a las asignaturas que imparte en la universidad, siendo una metodología educativa validada, confiable y acorde para el desarrollo de las competencias profesionales.

Referencias bibliográficas

Álvarez, J. M. (2008). Evaluar el aprendizaje en una enseñanza centrada en competencias. En Gimeno, J. (Comp.) Educar por competencias ¿qué hay de nuevo?, Madrid, Morata, pp.206-233. Dialnet métricas, 206-234. Obtenido de https://dialnet.unirioja.es/servlet/ articulo?codigo $=2752019$

Azevedo, D. A., Reis, J. D., Pinto, P., Pereira, C. M., Azevedo, C., \& Campos, E. L. (2017). Uso de la casuística en el proceso de enseñanza-aprendizaje de bioética en la salud. Revista bioét, $25(1), 82-88$.

Castillo, S., \& Cabrerizo, J. (2010). Evaluación educativa de aprendizajes y competencias. Madrid: Pearson educación, S.A.

Chomsky, N. (2013). El trabajo académico, el asalto neoliberal a las universidades y cómo debería ser la educación superior. Bajo el Volcán, 13(21), 121-134.

Coronel, M. A. (2007). Reflexiones sobre la evaluación de materias de humanidades. En B. C. Salinas, La evaluación de los estudiantes en la Educación Superior (págs. 23-29). Valencia: Universitat de Valencia.

España, J. N. (2017). La educación popular y el desarrollo de las organizaciones sociales. Revista Iberoamerican de producción académica y gestión educativa, 4(8), 1-26.

Ferrer, C. (2007). La evaluación en los laboratorios de física: una tradición renovada trasladable a otras materias. En B. \&. Salinas, La evaluación en los laboratorios de física: una tradición renovada trasladable a La evaluación de los estudiantes en la Educación Superior (págs. 30-36). Valencia, España: Universitat de Valencia.

Guanipa, L. (2017). Realidad socioeducativa universitaria desde la onto-epistemología a la luz de la gestión del conocimeinto. Revista Arjé, 12(22), 241-248.

Guerrero, C., \& Jiménez, M. L. (2011). Una visión estratégica de los recursos humannos en base a competencias. INCEPTUM, VI(10), 323 - 333.

Levi-Montalcini, R. (14 de Mayo de 2005). Vivimos dominados por impulsos de bajo nivel, como hace 50.000 años. El Pais. (E. González, Entrevistador) Obtenido de https://elpais.com/ diario/2005/05/15/domingo/1116129153_850215.html 
Moreno, T. (2012). La evaluación de competencias en educación. Sinéctica Revista electrónica de educación, 1-20.

Morín, E. (2011). introducción al pensamiento complejo. Barcelona, España: Gedisa.

Obando, J. A. (2014). Evaluación de competencias. Memorias, 12(22), 73-79.

OECD. (7 de Octubre de 2002). Organisation de Coopération et de Développement Economiques. Obtenido de OECD.org: https://www.oecd.org/education/skills-beyond-school/ definitionandselectionofcompetenciesdeseco.htm

Rojas, M. (2009). De la enseñanza basada en procesos mentales al neuroaprendizaje: evidencias biológicas. Ingeniería y Sociedad UC, 4(2), 74-82.

Salinas, B., Costillas, C., \& (Cood.). (2007). La evaluación de los estudiantes en la Educación Superior. Valencia, España: Universitat de Valencia.

SINEACE. (Octubre de 2016). SINEACE. Recuperado el 1 de Abril de 2020, de Sistema Nacional de Evaluacicón, Acreditación y Certificación de la Calidad Educativa: https://www.sineace. gob.pe/wp-content/uploads/2014/o8/Anexo-1-nuevo-modelo-programas-Resolucion-175. pdf

SINEACE. (Mayo de 2017). SINEACE. (S. P. Collado, Ed.) Recuperado el 31 de Marzo de 2020, de Sistema Nacional de Evaluación, Acreditación y Certificación de la Calidad Educativa: https://www.sineace.gob.pe/acreditacion/acreditacion-educacion-superior-universitaria/

Torres, A., \& Lugo, A. (2020). Hacia una nueva ciencia, desde la visión de Morín y Martínez Miguelez. Revista REDINE, 12(1), 37-43. 